\title{
LA INVESTIGACIÓN-ACCIÓN COMO ESTRATEGIA DE APRENDIZAJE EN LA FORMACIÓN INICIAL DEL PROFESORADO
}

\section{(*) Cristina Maciel de Oliveira}

SÍNTESIS: Los tres ejes de este artículo son: el profesor estratégico, el enfoque de investigación-acción en el marco de las teorías sobre la práctica de la función docente, y la investigación-acción como estrategia de aprendizaje en la formación del profesorado. Desde el discurso teórico interrelacionado con la experiencia de formadores en Investigación Educativa Aplicada, se plantean criterios de acción pedagógica para la formación inicial de profesionales de la enseñanza con habilidades regulativas para planificar, orientar y evaluar sus propios procesos cognitivos, en relación con los contenidos de aprendizaje a enseñar y con los vinculados a su actuación docente.

SÍNTESE: Os três eixos deste artigo são: o professor estratégico, o enfoque de investigação-ação no marco das teorias sobre a prática da função docente, e a investigação-ação como estratégia de aprendizagem na formação do professorado. A partir do discurso teórico inter-relacionado com a experiência de formadores em Investigação Educacional Aplicada, se determinam critérios de ação pedagógica para a formação inicial de profissionais do ensino com habilidades reguladoras para planificar, orientar e avaliar seus próprios processos cognitivos, em relação aos conteúdos de aprendizagem para ensinar e com os vinculados a sua atuação docente.

(*) Profesora de Investigación Educativa Aplicada, Centro Regional de Profesores del Este, Maldonado, Uruguay. 


\section{EL PROFESOR ESTRATÉGICO}

Las características de la actual sociedad y su incidencia en la educación (educación en el centro del debate público, reformas educativas, nuevas tecnologías, pérdida del monopolio de la información por las escuelas, por citar algunos factores), plantean importantes desafíos al docente en el desempeño de su rol, que deben ser atendidos desde su formación inicial.

No es casual el creciente número de trabajos de investigación sobre formación del profesorado publicados en Estados Unidos y en Europa en los últimos años, aunque en el contexto latinoamericano la problemática del profesor debutante en cuanto objeto de investigación es «cuasi-inexistente» según Cornejo (1999).

Los informes de las investigaciones que se desarrollan en este campo nos aportan respuestas a cuestiones que son medulares para constituir o para renovar los planes de estudio de formación inicial en la teoría y en la práctica de la enseñanza.

Una de esas cuestiones es: ¿cuál es el perfil de egreso que debe tener el profesor que hoy comienza a formarse como tal para educar y para enseñar de acuerdo con los desafíos que la sociedad le presenta? La línea de investigación de Monereo et al. (1998) sobre estrategias de aprendizaje en la universidad, nos aporta una posible respuesta a esta interrogante: el perfil de un profesor que asume los desafíos que le plantean los cambios sociales debe ser el de un profesional estratégico.

Monereo reconoce en la formación del profesorado una vía para enseñar estrategias de aprendizaje. Las define como:

[...] procesos de toma de decisiones (conscientes e intencionales) en los cuales el alumno elige y recupera, de manera coordinada, los conocimientos que necesita para cumplimentar una determinada demanda u objetivo, dependiendo de las características de la situación educativa en que se produce la acción (op. cit., p. 27).

El autor plantea la necesidad de pensar en una formación continua que tenga en cuenta al profesor como aprendiz y como enseñante estratégico, y que le aporte instrumentos para:

- Interpretar y analizar las situaciones profesionales en las que actúa. 
- Tomar decisiones como aprendiz y como docente estratégico que le permita enriquecer su formación.

En este sentido, conceptualiza al profesor estratégico (Monereo y Clariana, 1993, citado por Monereo et al., 1998) como un profesional con habilidades ${ }^{1}$ regulativas para planificar, orientar y evaluar sus propios procesos cognitivos, sean estos de aprendizaje de los contenidos a enseñar o sean relacionados con su actuación docente.

La idea de regulación es clave en el concepto de estrategia para estos autores. Implica reflexión consciente y control permanente del proceso de aprendizaje (planificación, realización de la tarea, evaluación de la propia conducta).

La aplicación consciente del sistema de regulación origina un tercer tipo de conocimiento, el condicional o estratégico. Éste surge de analizar las condiciones que determinan que una estrategia sea adecua$\mathrm{da}$, y permite establecer relaciones con ciertas formas de pensamiento y de acción. La actuación estratégica se realizaría según el conocimiento condicional que el sujeto había construido para esa situación, o que había actualizado en el caso de que las circunstancias fueran similares a las de una situación anterior en la que se hubiera utilizado eficazmente la estrategia.

El aprendizaje mediante estrategias, es decir, a través de la toma consciente de decisiones, promueve el aprendizaje significativo (en el sentido de Ausubel, 1963) puesto que no se trata sólo de aprender a utilizar procedimientos, sino a valorar las condiciones de su utilización y su efecto en el proceso de resolución de la tarea.

Por lo expuesto, entendemos que la investigación-acción puede ser considerada como una valiosa estrategia de aprendizaje en el nivel de formación docente inicial. Una estrategia sofisticada, puesto que es preciso que se enseñe de forma explícita (Monereo et al. 1998). Nuestra experiencia pedagógica en relación con esta modalidad de investigación nos permite afirmar que potencia la conciencia del estudiante de

${ }^{1}$ Monereo et al. (op. cit., p. 18) toman el concepto de «habilidades» de Sckmeck (1988) como «capacidades que pueden expresarse en conductas en cualquier momento, porque han sido desarrolladas a través de la práctica (es decir, mediante el uso de procedimientos) y que, además, pueden utilizarse o ponerse en juego, tanto consciente como inconscientemente, de forma automática». 
profesorado que tiene grupos de alumnos a cargo sobre cómo desarrollar y optimizar su quehacer docente, siendo a la vez aprendiz y profesor de la materia a enseñar.

\section{EL ENFOQUE DE INVESTIGACIÓN-ACCIÓN EN EL MARCO DE LAS TEORÍAS SOBRE LA PRÁCTICA DE LA FUNCIÓN DOCENTE Y LA FORMACIÓN DEL PROFESORADO}

Un breve análisis de las teorías que han prevalecido en los últimos treinta años sobre la práctica de la función docente y sobre la formación del profesorado nos permite centrar la atención en sus diversos enfoques, y re-significar aquel que hemos adoptado en nuestra práctica como formadores de profesores de enseñanza media: el de investigaciónacción.

Pérez Gómez (2000) propone una clasificación en la que distingue las siguientes cuatro perspectivas: académica, técnica, práctica, y de reflexión en la práctica para la reconstrucción social. Dicha propuesta surge de los aportes de Feiman-Nesmer y de Zeichner, ambos en 1990. Zeichner -citado por Pérez Gómez- afirma que las tres perspectivas ideológicas ${ }^{2}$ que han estado en conflicto en la mayor parte de los programas de formación docente son: la tradicional, según la cual la enseñanza es una actividad artesanal y el docente un artesano; la técnica, que entiende que la enseñanza es ciencia aplicada y el docente un técnico; y la radical, para la que la enseñanza es una actividad crítica y el docente un profesional que investiga reflexionando sobre su práctica.

En una línea de tiempo, se muestra en el cuadro 1 la evolución en el análisis de las perspectivas teóricas que han predominado en la práctica de la función docente y en la formación del profesorado en el último tercio del siglo XX.

La clasificación que ofrece Pérez Gómez particulariza las anteriores con la identificación de enfoques diferenciados en cada una de las perspectivas. Se presentan en el cuadro 2.

\footnotetext{
${ }^{2}$ Estas perspectivas fueron identificadas por Kirk (1986), quien se basó, a su vez, en la distinción de van Mannen (1977).
} 


\section{CUADRO 1}

Perspectivas ideológicas dominantes en el discurso teórico, en la práctica de la función docente y en la formación del profesorado en el último tercio del siglo XX, según Pérez Gómez (2000)

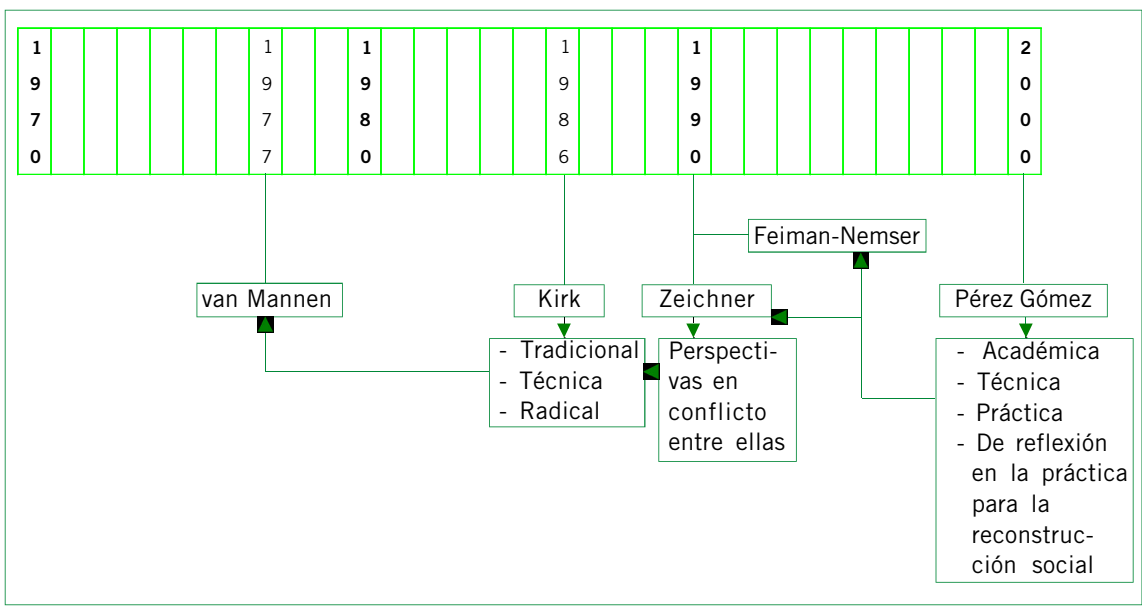

\section{CUADRO 2}

Perspectivas y enfoques sobre la función y la formación del profesor según Pérez Gómez (2000)

\begin{tabular}{|c|c|c|}
\hline Perspectivas & \multicolumn{2}{|c|}{ Enfoques } \\
\hline Perspectiva académica & Enfoque enciclopédico & Enfoque comprensivo \\
\hline Perspectiva técnica & $\begin{array}{c}\text { Modelo de } \\
\text { entrenamiento }\end{array}$ & $\begin{array}{c}\text { Modelo de adopción } \\
\text { de decisiones }\end{array}$ \\
\hline Perspectiva práctica & Enfoque tradicional & $\begin{array}{c}\text { Enfoque reflexivo } \\
\text { sobre la práctica }\end{array}$ \\
\hline $\begin{array}{c}\text { Perspectiva de reflexión } \\
\text { en la práctica para la } \\
\text { reconstrucción social }\end{array}$ & $\begin{array}{c}\text { Enfoque de crítica y } \\
\text { reconstrucción social }\end{array}$ & $\begin{array}{c}\text { Enfoque de } \\
\text { investigación-acción y } \\
\text { formación del profesor } \\
\text { para la comprensión }\end{array}$ \\
\hline
\end{tabular}


Siguiendo el análisis de Pérez Gómez, planteamos las características principales de cada una de estas perspectivas con sus correspondientes enfoques:

- La perspectiva académica en la formación del profesorado pone el acento en la transmisión de los conocimientos y en la adquisición de la cultura. El docente es el especialista que domina alguna de las disciplinas culturales, y su formación radica en el dominio de los contenidos que debe transmitir. Dentro de la perspectiva académica el autor diferencia los enfoques enciclopédico y comprensivo. El enciclopédico entiende la formación del profesor como un acopio de productos culturales que deberá exponer en su tarea docente con claridad y orden. El comprensivo busca desarrollar en el alumno la comprensión de la disciplina. El docente debe formarse en la epistemología de la misma y en la filosofía de la ciencia en general, además de integrar conocimientos didácticos referentes a la disciplina que enseña para su eficaz transmisión.

- La perspectiva técnica otorga a la enseñanza el atributo de ciencia aplicada, y valora la calidad de la misma en función de los productos logrados y de la eficacia para alcanzarlos. El profesor es un técnico cuya actividad se orienta sobre todo a la aplicación de teorías y de técnicas en la solución de problemas. En la visión de Pérez Gómez, dicha concepción es la que Schön (1983) denomina "racionalidad técnica», como epistemología de la práctica. Ha prevalecido a lo largo del siglo, particularmente en los últimos treinta años, en los procesos de enseñanza concebidos como pura intervención tecnológica, y en la investigación sobre la enseñanza enmarcada en el paradigma proceso-producto. En una valoración de esta postura, Pérez Gómez señala que la misma ha implicado un avance sobre el enfoque tradicionalista, artesanal y academicista, al entender que la enseñanza puede ser explicada con rigurosidad, sistematización y objetividad; a pesar de haberlo intentado durante las últimas décadas, «la tecnología educativa no puede afrontar las cada día más evidentes características de los fenómenos prácticos: complejidad, incertidumbre, inestabilidad, singularidad y conflicto de valores» (op. cit., p. 407). 
En la formación del docente, según la perspectiva técnica, el autor distingue dos modelos: el de entrenamiento y el de adopción de decisiones. La diferencia se encuentra en que el primero entrena al profesor en la aplicación de aquellas técnicas, procedimientos y habilidades que han demostrado su eficacia en investigaciones anteriores, en tanto que el segundo requiere la formación de competencias estratégicas que le posibiliten la adopción de decisiones adecuadas a partir de razonamientos basados en principios y en procedimientos de intervención.

- La perspectiva práctica entiende que la enseñanza es una actividad compleja, en la cual el contexto juega un rol determinante como creador de situaciones de conflicto de valor, que, en su mayoría, son imprevisibles y que demandan opciones éticas y políticas del docente. La formación del profesor dentro de esta perspectiva considera la práctica como principio y fin del aprendizaje, y al profesor experimentado como el recurso más eficaz para que el docente en formación desarrolle sus propias experiencias. Esta perspectiva ha evolucionado durante el siglo XX, distinguiéndose en ella el enfoque tradicional, que se basa casi exclusivamente en la experiencia práctica, y el enfoque que subraya la práctica reflexiva. El tradicional concibe la enseñanza como una actividad artesanal, que se aprende en la institución educativa en un lento proceso de inducción y de socialización. Basándose en investigaciones sobre el pensamiento pedagógico de los docentes novatos (Pérez Gómez y Gimeno, 1986; Pérez Gómez y Barquin, 1991), Pérez Gómez nos advierte sobre la incidencia que tiene en el profesor novato la vivencia institucional de los primeros años, en la medida en que ésta puede empobrecer el acervo teórico alcanzado durante su formación académica inicial. El enfoque reflexivo sobre la práctica reconoce la necesidad del docente de analizar y de comprender la complejidad de las situaciones áulicas e institucionales de las cuales forma parte. Schön analiza el conocimiento práctico como un proceso de reflexión en la acción. Sobre el concepto de reflexión, Pérez Gómez (op. cit., p. 417) afirma:

La reflexión implica la inmersión consciente del hombre en el mundo de su experiencia, un mundo cargado de 
connotaciones, valores, intercambios simbólicos, correspondencias afectivas, intereses sociales y escenarios políticos. La reflexión, a diferencia de otras formas de conocimiento, supone tanto un sistemático esfuerzo de análisis, como la necesidad de elaborar una propuesta totalizadora, que captura y orienta la acción.

El mismo autor distingue, de acuerdo con Schön (1983), los siguientes conceptos que integran la concepción más amplia de pensamiento práctico del profesional ante las situaciones de la práctica:

- Conocimiento en la acción, que se manifiesta en el saber hacer.

- Reflexión en la acción, que implica pensar sobre lo que se hace a la vez que se actúa, es decir, un metaconocimiento en la acción.

- Reflexión sobre la acción y sobre la reflexión en la acción, como el análisis que el sujeto realiza sobre la propia acción después de haberla hecho.

- En la perspectiva de reflexión en la práctica para la reconstrucción social, Pérez Gómez agrupa las posturas que conciben la enseñanza como una actividad crítica, social, con opciones de carácter ético. Distingue el enfoque de crítica y reconstrucción social del enfoque de investigación-acción y formación del profesorado para la comprensión. El enfoque de crítica y reconstrucción social reconoce en la escuela y en la formación del profesor los medios fundamentales para el logro de una sociedad más justa. La formación del profesor busca crear conciencia para pensar críticamente sobre el orden social de su comunidad. El profesor es un intelectual, un transformador de la sociedad comprometido políticamente. Representantes de este enfoque son: Giroux, Smith, Zeichner, Apple, Kemmis. En el enfoque de investigaciónacción y formación de/ profesorado para la comprensión:

[...] la práctica profesional del docente es considerada como una práctica intelectual y autónoma, no meramente técnica; es un proceso de acción y de reflexión cooperativa, de indagación y experimentación, donde el profesor/a aprende al enseñar y enseña porque aprende, interviene para facilitar y no imponer ni sustituir la comprensión de los alumnos/as, la reconstrucción de su conocimiento 
experiencial; y al reflexionar sobre su intervención ejerce y desarrolla su propia comprensión. Los centros educativos se transforman así en centros de desarrollo profesional del docente (Pérez Gómez, op. cit., p. 429).

Este enfoque tiene como principales representantes a Stenhouse, McDonald y Elliott.

\section{LA INVESTIGACIÓN-ACCIÓN COMO ESTRATEGIA DE APRENDIZAJE EN LA FORMACIÓN DEL PROFESORADO}

Es en el marco del enfoque de investigación-acción y formación del profesorado para la comprensión en el cual orientamos el curso de Investigación Educativa Aplicada ${ }^{3}$, correspondiente al Programa de Estudios de 3. er $^{2}$ ño de la carrera de formación de profesores para la educación media que se desarrolla en los centros regionales de profesores en Uruguay.

Hemos optado por dar prioridad a este enfoque, dado que la asignatura Investigación Educativa Aplicada:

[...] se sustenta en la necesidad de contar con una formación básica en investigación, que permite al estudiante discutir conceptual y operativamente las condicionantes y las formas de generar conocimiento a partir de la utilización del método científico, así como aplicar técnicas de investigación en diagnosticar problemas y desarrollar estrategias de intervención (Secretaría de Capacitación Docente, 2002).

Interesa la diferenciación de enfoques que establece Pérez Gómez dentro de la perspectiva de reflexión en la práctica para la reconstrucción social, puesto que en nuestra experiencia pedagógica de formación de profesores ponemos el acento en la reflexión en y sobre la práctica docente y en la transformación de ella que puedan realizar los profesores que en la reconstrucción social, aunque sin dejar de animarlos a reconocer el papel que pueden adoptar en su entorno social como intelectuales capaces de pensarlo críticamente, y aún de transformarlo si lo estiman conveniente.

${ }^{3}$ Este curso se desarrolló de marzo a diciembre de 2003, con una carga horaria semanal de 4 horas. 
Observar de manera sistemática las situaciones educativas (Postic y de Ketele, 1992) de las cuales forman parte como profesores (tanto áulicas como institucionales) y analizarlas con fundamentos teóricos, elaborar planes de intervención e implementarlos, evaluarlos y formular informes de investigación, reelaborar dichos planes, trabajar de modo colaborativo, comunicar públicamente los procesos de investigación desarrollados, son las principales actividades sobre las que estructuramos la modalidad de investigación-acción como estrategia de formación.

Esta estrategia de aprendizaje no se enseña al comienzo del curso. Es un componente vinculante del programa de estudios, que se inicia en el primer semestre con la construcción de la dimensión ética por el profesor investigador en su etapa de formación inicial, para que sobre la base de la reflexión y el discernimiento de principios y valores que sustentan la acción de investigar, ésta se desarrolle con actitud responsable sobre sus posibles consecuencias en los sujetos, en fenómenos y en situaciones de investigación.

El estudio de la observación científica y de sus características, y el trabajo consciente de los estudiantes de profesorado con sus capacidades de ver y de oír sobre situaciones educativas de las que forman parte para llegar a transformarse en observadores con cierto grado de habilidad, son otros pilares del curso para la aplicación de los procedimientos de investigación (observación, entrevista, encuesta y sociometría), con el propósito de ampliar y sistematizar el conocimiento de dichas situaciones.

El análisis de los atributos de los paradigmas cuantitativo y cualitativo y sus interrelaciones con las metodologías de análisis de datos, la comparación entre las tendencias contemporáneas en investigación educativa y los diversos modos de acercarse a la realidad [positivista, interpretativo y crítico (Pérez Serrano, 1990)], son parte del marco teórico que ilumina la libre opción por parte de los estudiantes de profesorado (profesores practicantes), y de la finalidad que guiará su trabajo de investigación en el segundo y último semestre del curso: ¿describir la situación educativa elegida? ¿interpretarla desde la perspectiva de los sujetos que intervienen en ella? ¿describirla e interpretarla? ¿transformar dicha situación empleando metodología profesional a partir de su descripción e interpretación?

Ante la necesidad que tienen los estudiantes de profesorado de resolver situaciones problemáticas de su novel práctica docente, el 
enfoque de investigación-acción emerge como opción teórica y metodológica para investigarlas y transformarlas.

Para desarrollar un proceso de esta naturaleza seguimos las siguientes fases, en una adaptación de la guía práctica de Elliott (1996), que en función de nuestra experiencia construimos en 2002 (Maciel de Oliveira, 2003):

- Identificación del área problemática de la práctica docente.

- Organización del equipo de trabajo.

- Exploración de la situación inicial mediante el uso de procedimientos de investigación y el estudio teórico de la temática.

- Planteo de conclusiones en informe escrito y puesta oral en común.

- Enunciado del problema y formulación de objetivos.

- Identificación de factores a modificar y planteo de hipótesis-acción ${ }^{4}$.

- Planificación de estrategias a desarrollar.

- Aplicación de estrategias y valoración de su impacto mediante la aplicación de instrumentos de investigación.

- Planteo de reflexiones y conclusiones.

- Revisión del plan general y replanteo de hipótesis-acción.

- Nuevas observaciones, acciones y reflexiones.

- Comunicación pública del proceso realizado.

Las situaciones problemáticas identificadas en el Liceo de Práctica ${ }^{5}$ que se estudiaron siguiendo la modalidad de investigaciónacción en el curso 2003, fueron:

${ }^{4}$ La hipótesis-acción «indica una acción a realizar que debe responder, sobre todo, a una autorreflexión y autocomprensión de la situación. La hipótesis nace de la reflexión y del análisis de la problemática, tanto en el plano teórico como en el práctico» (Pérez Serrano, op. cit., p.107).

${ }^{5}$ Liceo n. ${ }^{\circ} 4$ de Maldonado, en el que realizaron su práctica docente en 2003 los 70 estudiantes de profesorado del curso 2001, que siguen la carrera en el Centro Regional de Profesores del Este, Maldonado. 
- Énfasis que dan los profesores del Liceo de Práctica a la adquisición de contenidos procedimentales, en relación con los contenidos conceptuales y actitudinales.

- Perspectiva de una nueva experiencia en el Liceo de Práctica: el Consejo Estudiantil.

- Integración de la informática en la clase de Matemática.

- Instrumentos de evaluación en el aula.

- La resolución de problemas: un enfoque desde el pensamiento lógico.

- Construcción del rol docente: analizar y criticar la práctica docente.

- Planificación diaria.

- La formulación de preguntas en el aula de Ciencias Sociales por parte de los profesores practicantes.

- Integración de los estudiantes de otros liceos al proyecto educativo de centro del Liceo de Práctica.

- La articulación de los distintos contenidos dentro del aula de Ciencias Sociales.

A continuación presentamos dos de estas experiencias. Organizamos la síntesis de los informes de cada equipo de trabajo en las dimensiones profesor aprendiz y profesor enseñante, en el entendido de que pueden ilustrar el proceso de investigación-acción que orientamos como estrategia de aprendizaje en procura de la formación de profesores estratégicos.

3.1 SIINTESISDELAINVESTIGACIÓN-ACCIÓNREALIZADASOBREFORMULACIÓN DE PREGUNTAS ORALES EN EL AULA POR PROFESORES PRACTICANTES DE CIENCIAS SOCIALES ${ }^{6}$, EN TANTO APRENDICES Y ENSEÑANTES

\section{PROFESOR APRENDIZ}

Del área disciplinar

- Ciencias Sociales.

${ }^{6}$ Profesores practicantes: Patricia Astessiano, Analía Chiarle, Ana Luisa Loyarte, Sabina Maneiro, Eduardo Meroni, Delia Prochazka. 
De teorías

- Aportes de la Psicología de la Educación a las técnicas de enseñanza y de aprendizaje de las Ciencias Sociales.

- Enfoque constructivista.

- Relación docente-alumno en el aula.

- Las preguntas orales en clase: funciones, tipos de preguntas, orientaciones.

De procedimientos de investigación

- Observación de la formulación de preguntas orales en clase (1. ${ }^{\circ}$ y $2 .^{\circ}$ año de Ciencias Sociales y $3 .^{\circ}$ de Geografía).

- Entrevistas a profesores tutores de práctica docente sobre la formulación de preguntas por los profesores practicantes en el desarrollo de sus clases.

\section{De formulación de hipótesis}

- La correcta formulación de las preguntas en el aula tiene relación con la mejora en la calidad de la transposición didáctica de la materia y con el mejoramiento del proceso de enseñanza-aprendizaje de los alumnos.

\section{PROFESOR ENSEÑANTE}

\section{Identificación de la situación problema}

- Formulación de preguntas orales en el aula por parte de los profesores practicantes. De las observaciones realizadas con carácter diagnóstico se concluye que la mayoría de los profesores practicantes utiliza las preguntas en el aula para evaluar. Las orientan hacia sí mismos en más oportunidades que hacia los alumnos. Tienden a responder ellos mismos inmediatamente. Las preguntas propenden a ser memorísticas o de contenido factual. Casi no se observan preguntas reflexivas, de estímulo para el debate, de análisis, o que exijan algún tipo de ejemplificación por los alumnos. Existe relación entre lo manifestado por los profesores tutores y las observaciones realizadas: la mayor parte de las preguntas orales que no se planifican antes son formuladas de forma incorrecta. 


\section{Desarrollo de habilidades regulativas}

- Ser consciente de los objetivos:

Generales

- Mejorar la práctica docente a través de la correcta formulación de preguntas orales y de la relación entre éstas y los objetivos de la clase.

- Disminuir las dificultades de los profesores practicantes en la formulación de las preguntas orales en clase.

\section{Específicos}

- Reconocer las dificultades reales en la formulación de preguntas orales en clase por parte de los profesores practicantes.

- Identificar posibles formas para mejorar esta situación.

- Diseñar indicadores para la formulación de preguntas orales.

- Proponer estrategias de planificación, teniendo en cuenta los indicadores utilizados para la correcta formulación de las preguntas.

- Formular preguntas relacionadas con los objetivos y con los contenidos conceptuales, procedimentales y actitudinales.

- Plantear la hipótesis-acción:

- La planificación de las preguntas orales en relación con los objetivos de la clase mejora la conducción de la misma por el profesor, y el aprendizaje de los contenidos por los alumnos.

- Planificación de las estrategias:

- Planificación de las preguntas a realizar.

- Considerar los objetivos de la clase en la planificación de las preguntas y en el tipo de preguntas a formular (para motivar, para indagar ideas previas, de evaluación, inicio, proceso o cierre de la clase, cerradas, semicerradas o abiertas, de argumentación, etc.). 
- Observar y registrar en una planilla la formulación de preguntas durante dos semanas.

- Aplicación de las estrategias y control del curso de acción.

- Evaluación de la actuación.

\section{Toma consciente de decisiones}

- Proponer instancias de autoevaluación y de observación de la propia práctica en relación con la formulación de preguntas.

- Informar al equipo docente del liceo sobre la propuesta, los logros y las dificultades de la misma.

\section{Reflexión sobre la experiencia}

- La planificación de las preguntas no puede ser impuesta; es responsabilidad de cada profesor. Esto se relaciona con la profesionalización y con la ética docente.

- La planificación de las preguntas, teniendo en cuenta los objetivos de la clase, ha evidenciado una relación con el aprendizaje significativo de los alumnos.

- Una de las principales causas de la carencia de planificación de las preguntas se relaciona con la falta de tiempo real que se destina a la planificación de la clase.

- La planificación de las preguntas estructura el hilo conductor de la clase, y permite al profesor un manejo adecuado del tiempo de clase y de la secuencia didáctica.

\section{Idea general revisada para la orientación de un nuevo ciclo} de acción

- La planificación excesiva de las preguntas puede quitar flexibilidad al profesor en la conducción de la clase. 
3.2 SIINTESISDELAINVESTIGACIÓN-ACCIÓNREALIZADA SOBREINTEGRACIÓN DE LA INFORMÁTICA EN LA CLASE DE MATEMÁTICA POR PROFESORES PRACTICANTES DE MATEMÁTICA', EN TANTO APRENDICES Y ENSEÑANTES

\section{PROFESOR APRENDIZ}

Del área disciplinar

- Matemática.

De teorías

- Práctica docente.

- Motivación.

- Nuevas tecnologías como recurso didáctico.

- Informática educativa.

- Aprendizaje autónomo.

- Autoestima.

De procedimientos de investigación

- Entrevista informal a profesora coordinadora del Departamento de Informática del Liceo de Práctica sobre el posible uso del laboratorio de informática en los horarios correspondientes a la clase de Matemática.

- Encuesta a los alumnos sobre el gusto por el uso de la computadora, frecuencia de uso fuera del liceo y programas que maneja.

De formulación de hipótesis

- La informática es un medio didáctico motivador del aprendizaje de la Matemática por los alumnos.

${ }^{7}$ Profesoras practicantes: Alejandra Cevallos, Elena Domínguez, Paula García, Graziella Nucciotti, Ana Laura Núñez y Carolina Sastre. 


\section{PROFESOR ENSENANANE}

\section{Identificación de la situación problema}

- La actitud pasiva de algunos alumnos en la clase de Matemática y la necesidad de favorecer su participación nos orienta a buscar nuevos medios didácticos como la informática.

\section{Desarrollo de habilidades regulativas}

- Ser consciente de los objetivos:

Generales

- Mejorar la práctica docente incorporando la informática como medio didáctico.

- Favorecer la participación de los alumnos de $1 .^{\circ}(5)$ y $1 .^{\circ}$ (6) en la clase de Matemática, estimulando su creatividad y su aprendizaje autónomo.

\section{Específicos}

- Facilitar la construcción y la visualización de figuras geométricas de los alumnos de $1 . .^{\circ}(5)$ y $1 . .^{\circ}(6)$ mediante eluso de un software de geometría.

- Plantear la hipótesis-acción.

- El uso del software de geometría facilitará la construcción y la visualización de figuras geométricas.

- Planificación de las estrategias:

- Coordinar horarios para asistir al laboratorio de informática.

- Escoger el software e instalarlo en las computadoras.

- Planificar secuencia de trabajo guiada a realizar en el laboratorio de informática con el software de geometría.

- Aplicación de las estrategias y control del curso de acción.

- Observación participante de aspectos procedimentales y actitudinales con planilla de observación, y encuesta a alumnos para conocer si les gustaría volver a usar el laboratorio de informática para la clase de Matemática, si la tarea 
con el software les facilita o no la construcción de figuras geométricas, y si les favorece trabajar con un compañero.

- Evaluación de la actuación.

\section{Toma consciente de decisiones}

- Adoptar la Informática como un nuevo medio didáctico para motivar el aprendizaje de la Matemática por los alumnos.

- Buscar software apropiado y planificar actividades para su uso.

- Informar al equipo docente del liceo sobre la propuesta, los logros y las dificultades de la misma.

\section{Reflexión sobre la experiencia}

- La hipótesis se cumplió tanto en lo concerniente al logro de una mayor motivación de los alumnos, como en facilitar la construcción y la visualización de características de figuras geométricas. Además, se vio favorecido el trabajo con el compañero.

- El uso del software requiere una cuidadosa planificación del trabajo guiado.

\section{Idea general revisada para la orientación de un nuevo ciclo de acción}

- Sería conveniente planificar el uso del software no sólo para verificar propiedades geométricas, sino también para promover el descubrimiento y la visualización geométrica de figuras, movimientos y lugares geométricos con sus respectivas características.

\section{BIBLIOGRAFÍA}

CORNEJO ABARCA, José (1999): "Profesores que se inician en la docencia: algunas reflexiones al respecto desde América Latina», en Revista Iberoamericana de Educación, núm. 19, enero-abril, Madrid, OEl. 
ELLIOTT, John (1996): El cambio educativo desde la investigación-acción, 2. ${ }^{\text {a }}$ ed., Madrid, Morata.

MACIEL DE OLIVEIRA, Cristina (2003): «Investigar, reflexionar y actuar en la práctica docente», en Revista Iberoamericana de Educación, versión digital, julio, Madrid, OEI, <http://www.campus-oei.org/revista/frame_lectores1.htm>.

\section{SECCIÓN INVESTIGACIÓN EDUCATIVA}

MONEREO, Carles (coord.) et al. (1998): Estrategias de enseñanza yaprendizaje, 5. ${ }^{a}$ ed., Barcelona, Graó.

PÉREZ GómEZ, Ángel (2000): «Capítulo XI. La función y formación del profesor en la enseñanza para la comprensión. Diferentes perspectivas», en José Gimeno Sacristán y Ángel Pérez Gómez, Comprender y transformar la enseñanza, 9. ${ }^{a}$ ed., Madrid, Morata. Madrid, Dykinson.

Pérez SerRano, Gloria (1990): Aplicaciones al campo social y educativo,

Postic, Marcel y KETELE, Jean-Marie de (1992): Observar las situaciones educativas, Madrid, Narcea.

SECRETARÍA de CAPACITACIÓn DOCENTE (2000): Programa de 3 . $^{\text {er }}$ año de Investigación Educativa Aplicada, Inédito. 


\title{
Contactar
}

Revista lberoamericana de Educación

\author{
Principal OEI
}

